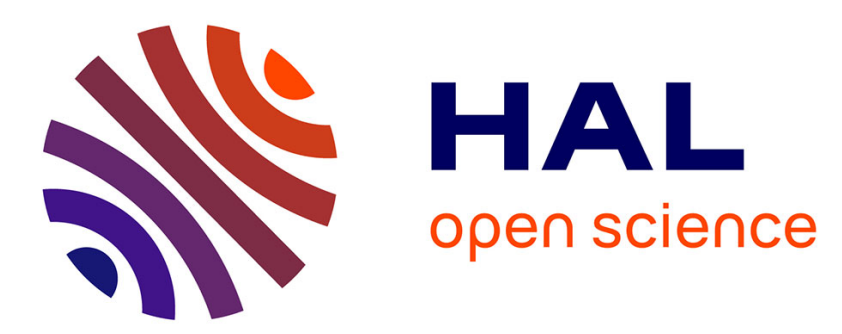

\title{
Determination of the Coulombic Interaction Parameters of the Extended Hubbard Model in the Organic Conductors
}

\author{
F. Castet, Agnès Fritsch, L. Ducasse
}

\section{- To cite this version:}

F. Castet, Agnès Fritsch, L. Ducasse. Determination of the Coulombic Interaction Parameters of the Extended Hubbard Model in the Organic Conductors. Journal de Physique I, 1996, 6 (4), pp.583-597. 10.1051/jp1:1996231 . jpa-00247204

\section{HAL Id: jpa-00247204 https://hal.science/jpa-00247204}

Submitted on 1 Jan 1996

HAL is a multi-disciplinary open access archive for the deposit and dissemination of scientific research documents, whether they are published or not. The documents may come from teaching and research institutions in France or abroad, or from public or private research centers.
L'archive ouverte pluridisciplinaire HAL, est destinée au dépôt et à la diffusion de documents scientifiques de niveau recherche, publiés ou non, émanant des établissements d'enseignement et de recherche français ou étrangers, des laboratoires publics ou privés. 


\title{
Determination of the Coulombic Interaction Parameters of the Extended Hubbard Model in the Organic Conductors
}

\author{
F. Castet, A. Fritsch $\left(^{*}\right)$ and L. Ducasse $\left(^{*}\right)$ \\ Laboratoire de Physico-Chimie Théorique $\left({ }^{* *}\right)$, Université de Bordeaux I, 33405 Talence Cedex, \\ France
}

(Received 23 October 1995, received in final form and accepted 20 December 1995)

PACS.31.20.Rx - Valence bond calculations ( $a b$ inttio or not)

PACS.71.10.+x - General theories and computational techniques

PACS.71.28.+d - Narrow-band systems: heavy-fermion solids; intermediate-valence solids

\begin{abstract}
A mixed Valence-Bond/Hartree-Fock method applied to all valence electrons calculations on finite size clusters was derived in order to size up the relative magnitude of the coulombic interactions between conduction electrons in organic conductors. An effective Hamiltonian built upon a single site orbital per molecule reproduces the whole set of VB energy levels. This extraction is most consistent when molecular orbitals optimized on monomers are used. Coulombic parameters describing the interactions in clusters of organıc molecules derived of TTF (tetrathiafulvalene) may be thus extracted. The magnitude of the coulombic terms $(U \approx 4 \mathrm{eV} ; V$ (nearest-neighbour) $=2-3 \mathrm{eV}$ ) differs qualitatively from previous estimations and evidences the long range part of the potential. The calculated charge transfer excitations are still in good agreement with the experimental results.

Résumé. - Une méthode mixte Valence-Bond/Hartree-Fock appliquée à des calculs tous électrons de valence sur des agrégats de taille finie a été développée afin de déterminer l'ordre de grandeur des interactions coulombiennes entre électrons de conduction dans les conducteurs organiques. Un Hamiltonien effectif construit dans la base d'une seule orbitale de site par molécule reproduit l'ensemble des niveaux d'énergie VB. Cette extraction est la plus cohérente lorsque I'on utilise des orbitales moléculaires optimisées sur les monomères. Les paramètres de Coulomb quı décrivent les ınteractions dans les agrégats de molécules organiques dérıvées du TTF (tétrathiofulvalène) ont ainsı pu être obtenus. Les termes coulombiens ( $U \approx 4 \mathrm{eV} ; V$ (premiers voısıns) $=2-3 \mathrm{eV}$ ) diffèrent qualitativement des estimations antérieures et mettent en évıdence la partie longue portée du potentiel. Les excitations à transfert de charge calculées demeurent cependant en bon accord avec l'expérience.
\end{abstract}

\section{Introduction}

Since the discovery of organic superconductivity in the Bechgaard salts TMTSF 2 X (TMTSF $=$ tetramethyltetraselenafulvalene; $\mathrm{X}^{-}$anion) [1], the maximum critical temperature $T_{c}$ was raised to $12.8 \mathrm{~K}$ [2]. Meanwhile, the true origin of this property (BCS or non-BCS mechanism, microscopic nature of the screening of the electron-electron repulsion) remains unclear. The

$\left({ }^{*}\right)$ Authors for correspondence (e-mall: ducasse@lpct.u-bordeaux.fr)

$\left({ }^{* *}\right)$ URA 503 CNRS

(C) Les Éditions de Physıque 1996 
superconductivity is not the single property to which a large literature has been devoted in the past few years [3]. The phase diagram of monodimensional (1D) or quasi-1D salts, represented by the Bechgaard salts $\mathrm{TMTSF}_{2} \mathrm{X}$ [4] and their sulfur analogs TMTTF $2 \mathrm{X}$ (TMTTF = tetramethyl-tetrathiafulvalene) [5], is complex: insulator (antiferromagnetic, AF; non magnetic, spin-Peierls, SP), metallic or superconducting states have been observed. More 2D salts, as those obtained from the derivatives of the BEDTTTF molecule (bisethylenedithiatetrathiafulvalene), also show a competition between magnetic and superconducting ground states: this is for example met in the $\kappa-(\mathrm{BEDTTTF})_{2} \mathrm{CuN}(\mathrm{CN})_{2} \mathrm{Cl}$ salt [6].

The difficulty in a theoretical analysis of these salts lies in their complex microscopic structure (one, two or more large molecules per unit cell, partial filling of the one-electron band resulting from the transfer between the anion and the cation, . .) and on the possible interplay between various terms (kinetic part of the Hamiltonian, Coulomb interaction, electron-phonon coupling, disorder of the anion). Among these factors, the electronic correlations certainly appear to be a leading term. For example, the limitation of the one-electron model is clearly seen in the band structure results on the $\mathrm{M}_{2} \mathrm{X}$ salts. This approach leads to a metallic behavior for every kind of salt based on the unit formula $\mathrm{M}_{2}^{+} \mathrm{X}^{-}$, although the conductivity measurements show metallic or semi-conducting behaviors for salts exhibiting very similar crystal structures [7].

The effects of the electron-electron correlations have been investigated using different theoretical models. The first one is the Ferm1 gas model also known as the g-ology model [8,9], derived in the weak coupling limit where the correlation terms are considered as a perturbation to the one-electron formalism. Low-energy properties may be analyzed within this approach: phase transitions, localization processes and NMR relaxation times. In the opposite limit of strong coupling, the Hubbard Hamiltonian involves transfer integrals and potential terms which are large energy parameters. Moreover, Mila and Zotos [10] have shown recently that even the low-energy properties may be described within the framework of this Hamiltonian.

Local methods have been developed in an attempt to solve the Hubbard or extended Hubbard models for clusters of finite size using Monte Carlo simulations and Lanczos technique [10-12]. In the domain of organic salts, the solutions of such Hamiltonians have been investigated as a function of the delocalization $(t)$ and correlation parameters $(U, V)[10,12]$. The one-electron transfer terms may be obtained from tight-binding band structure calculations [13] but very few attempts have been reported for the correlation terms. In the domain of high- $T_{\mathrm{c}}$ oxides, such calculations have been reported [14] and it is also possible to extract useful information from the combination of photoemission and Auger spectrocopies. In the domain of organic conductors, the situation is not so favorable because, on one hand, the photoemission spectra is controversial and, on another hand, the fact that the active (molecular) site contains a large number of atoms constitutes a strong limitation for theoretical approaches.

Recently, we analyzed the solutions of the extended Hubbard Hamiltonian by using crude estimates of $U$ and $V$ terms describing the Bechgaard salts [12]. This paper is aimed to present a more accurate and consistent model to determine these terms from cluster calculations involving all valence electrons of the organic molecules.

\section{Method}

We adopt a local point of view in the spirit of the Valence Bond (VB) approach. Within this model, the wavefunctions are developed on a basis of multielectronic functions that can be associated with distinct charge transfer states. These configurations are defined by ascribing a given number of electrons on each molecule. This is achieved by imposing to each orbital which is involved in the electronic configuration to be developed on the atomic orbitals of a specific molecule. 
This approach is restricted to the valence electrons of the organic molecule and we used the semi-empirical AM1 Hamiltonian [15]. The aim of this work is to obtain some information on the relative magnitude of the electronic interactions so that it is not really necessary to perform sophisticated $a b$ initio calculations. Furthermore, Demiralp and Goddard [16] have shown that the semi-empirical MNDO energies and the ionization potential of the molecule and the cation of BEDT-TTF are similar to the corresponding $a b$ initio results.

Let us consider a cluster of $N$ molecules. In a given electronic configuration, the molecule $i$ bears $n_{\imath}$ electrons: $n_{\imath}$ takes only 3 values if one takes only into account the charged species $\mathrm{M}^{0}$, $\mathrm{M}^{+}$and $\mathrm{M}^{2+}$. The cluster electronic configuration is then built on a set of orthogonal molecular orbitals (MO) doubly or singly occupied. Each molecule may therefore be described by two electronic shells: the closed shell collecting all the doubly occupied MO's and the open shell which contains at most one MO, which is assumed to be the highest occupied MO (HOMO).

In such a configuration, the total electronic energy is thus expressed following the Restricted Open Shell Hartree-Fock (ROHF) formulation [17]:

$$
E=2 \operatorname{Tr}\left[R_{1}\left(h+\frac{1}{2} G_{1}\right)\right]+\operatorname{Tr}\left[R_{2}\left(h+\frac{1}{2} G_{2}\right)\right]
$$

where the index 1 (2) refers to the closed (open) shell, $R_{2}$ is the first order density matrix, $h$ is the one-electron Hamiltonian matrix (involving kinetic term as well as attractive terms between the core and the valence electrons). $G_{\imath}$ is the two-electron interaction matrix involving the Coulomb $J$ and exchange $K$ matrices. All matrices may be expressed in the atomic orbital basis. With M AO's per molecule, one is lead to $(N * M)^{2}$ matrices. One has (in atomic units):

$$
\begin{gathered}
G_{1}=G\left(2 R_{1}\right)+G\left(R_{2}\right) \quad \text { closed shell } \\
G_{2}=G\left(2 R_{1}\right)+G^{\prime}\left(R_{2}\right) \quad \text { open shell } \\
G(R)=J(R)-\frac{1}{2} K(R) \quad G^{\prime}(R)=J(R)-K(R) \\
J(R)_{r s}=\sum_{t, u} R_{u t}\left\langle r t\left|1 / r_{\mu v}\right| s u\right\rangle \quad K(R)_{r s}=\sum_{t, u} R_{u t}\left\langle r t\left|1 / r_{\mu v}\right| u s\right\rangle
\end{gathered}
$$

All atomic one and two electron integrals are evaluated from the crystallographic coordinates of the atoms, following the AM1 parametrization. This part of the calculation prevents us from using cyclic boundary conditions which would avold possible edge effects. We shall see below that a thorough analysis of the results on chain clusters nevertheless provides parameters which do not depend on the finite size of the clusters.

The restriction to strictly molecular orbitals combined with the AM1 approximations has several consequences :

- the density matrix is block-diagonal in the atomic orbital basis, had the AO's been classified according to the molecules' label. Such a bloc-diagonal form allows us to consider only the molecular blocks of all interaction matrices. Each molecule may thus be fully characterized by $M * M$ matrices,

- the total electronic energy is the sum of molecular energies,

- a convenient simplification furthermore arises from the semi-empirical nature of the AM1 integrals and the local nature of the MOs, that lead to the vanishing of all intermolecular exchange effects so that any configuration can be cast into a single Slater determinant 
with all unpaired electrons bearing the same spin without any effect on the energy. Discriminating between the different spin states would only arise from the interaction between the various configurations. Such a simplification also appears in the more approximate and phenomenological quantum cell (frontier orbital) models such as the Hückel, Hubbard or Pariser-Parr-Pople models.

2.1. Optimization of Each Electronic Configuration. - It is important to notice that the neglect of differential overlap involved in the AM1 approximation together with the purely molecular nature of the orbitals may also lead to a viable optimization procedure of the MOs in the spırit of the Hartree-Fock method. The energy functional may be optimized with respect to the atomic orbital coefficients subject to the normalization constraints upon the MOs. Two stationnary conditions are involved in this procedure: one for the closed shell MOs, the second one for the open shell one. This draws us to two distinct Fock equations relative to each shell:

$$
\begin{array}{ll}
F_{1} C_{1}=\varepsilon_{1} C_{1} ; & F_{1}=h+G_{1} \\
F_{2} C_{2}=\varepsilon_{2} C_{2} ; & F_{2}=h+G_{2}
\end{array}
$$

where the $F_{2}$ are the Fock matrices for the closed and open shells respectively, the $C_{\imath}$ are the eigenvectors and $\varepsilon_{2}$ the MO's energies. Only the molecular blocks are considered here so that we have in fact two equations per molecule, i.e. at most $2 N$ equations. These relations are valid only if we can insure that the orthogonality constraints between the closed and the open shell MOs are fulfilled. This is accomplished by conducting a two-step self-consistent field iterative process. First, starting from a set of guess MOs, the closed shell equation is solved, producing a new set of orbitals represented by the $C_{1}$ matrix. Secondly, the open shell Fock equation is projected into the virtual subspace spanned by the empty orbitals obtained from the closed shell equation. In this way, this projected equation produces new open shell orbitals (occupied or empty) which belong to the orthogonal complement of the closed shell. The procedure is repeated until convergence of the total electronic energy.

Such a calculation therefore provides us with optimized sets of MOs for each charge transfer configuration. In this way, we allow for the relaxation of the valence MOs of the molecules under the influence of their own charge, but also under that of the neighbouring molecules.

2.2. Extended Hubbard Hamiltonian. - The relative energies of the VB configurations used in the calculations may be tentatively expressed in terms of the electrostatic parameters entering the definition of the following extended Hubbard Hamiltonian:

$$
\begin{aligned}
H= & -\sum_{\imath, \jmath, \sigma} t_{\imath \jmath}\left(c_{\imath \sigma}^{+} c_{\imath+1, \sigma}+\text { h.c. }\right)+\frac{1}{2} \sum_{\imath} U_{\imath} n_{\imath}\left(n_{\imath}-1\right) \\
& +\frac{1}{2} \sum_{\imath \neq \jmath} V_{\imath \jmath}\left(n_{\imath}-Z_{\imath}\right)\left(n_{\jmath}-Z_{\jmath}\right)
\end{aligned}
$$

where $t_{\imath \jmath}$ are the transfer integrals restricted to first neighbours, $U_{\imath}$ are the on-site repulsion energies and $V_{\imath \jmath}$ are the electrostatic interaction terms (in the following, $i-j$ defines the $(i-\jmath)^{\text {th }}$ neighbour); $c_{\imath, \sigma}^{+}\left(c_{\imath}, \sigma\right)$ is a creation (annuhilation) operator for a site orbital located at site $i$ with spin $\sigma, n_{\imath, \sigma}=\left(C_{\imath, \sigma}^{+} c_{\imath, \sigma}\right)$ is the operator number of electrons. The $Z_{\imath}^{\prime}$ s are core charges, so that non zero terms only occur between $\mathrm{M}^{+}$and/or $\mathrm{M}^{2+}$ cations. It is noteworthy to remark that the total energies related to the Hamiltonian (4) are not equivalent to the energies deduced from the resolution of equation (3). Our aim is to look for a possible relationship between energy differences calculated within the two approaches. 
Table I. - Results on the neutral $\mathrm{TTF}^{0}$, monocationic $\mathrm{TTF}^{+}$and dicationic $\mathrm{TTF}^{2+}$ species: $E$ are the total energies, $I_{1 \mathrm{t}}\left(T T F^{0}\right)$ and $I_{1 \mathrm{~K}}\left(T T F^{0}\right)$ are the calculated first ionization potentials for $\mathrm{TTF}^{0}$ using total energy differences and Koopman's theorem respectively, and $I_{1 \mathrm{t}}\left(\mathrm{TTF}^{+}\right)$ and $I_{1 \mathrm{~K}}\left(T T F^{+}\right)$are the corresponding data for the charged molecule $\mathrm{TTF}^{+} U$ is relative to equation (5) (all entries in eV).

\begin{tabular}{|l|l|}
\hline & $\begin{array}{l}\text { total energies and } \\
\text { ionization potential }\end{array}$ \\
\hline$E\left(\mathrm{TTF}^{0}\right)$ & -1599.767 \\
\hline$E\left(\mathrm{TTF}^{+}\right)$ & -1592.806 \\
\hline$E\left(\mathrm{TTF}^{++}\right)$ & -1581.200 \\
\hline$I_{1 \mathrm{t}}\left(\mathrm{TTF}^{0}\right)$ & 6.97 \\
\hline$I_{1 \mathrm{~K}}\left(\mathrm{TTF}^{0}\right)$ & 7.28 \\
\hline$I_{1 \mathrm{t}}\left(\mathrm{TTF}^{+}\right)$ & 11.60 \\
\hline$I_{1 \mathrm{~K}}\left(\mathrm{TTF}^{+}\right)$ & 11.95 \\
\hline$U$ & 4.64 \\
\hline
\end{tabular}

\section{Results}

In order to check the reliability of the method, we first calculated the energies of model TTF (tetrathiafulvalene) regular chains (the interplanar distance between neighboring TTF is fixed to $3.5 \AA$ ).

3.1. Monomers. - Table I shows the total energies obtained for the neutral TTF ${ }^{0}$, monocationic $\mathrm{TTF}^{+}$and dicationic $\mathrm{TTF}^{2+}$ species. The monocation was treated by means of the ROHF approach.

The calculated first ionization potentials for $\mathrm{TTF}^{0}$, using total energy differences $\left(I_{1 \mathrm{t}}\left(\mathrm{TTF}^{0}\right)=\right.$ $6.97 \mathrm{eV})$ and Koopman's theorem $\left(I_{1 \mathrm{~K}}\left(\mathrm{TTF}^{0}\right)=7.28 \mathrm{eV}\right)$ are in good agreement, as usually found for closed-shell system. For the charged molecule $\mathrm{TTF}^{+}$, the same difference of $0.3 \mathrm{eV}$ appears between $I_{1 \mathrm{t}}\left(\mathrm{TTF}^{+}\right)=11.60 \mathrm{eV}$ and $I_{1 \mathrm{~K}}\left(\mathrm{TTF}^{+}\right)=11.95 \mathrm{eV}$. This agreement results from the adequacy of the method employed. This equality is indeed implicit in the true Restricted Open shell Hartree-Fock formalism ; which is not true for the widely used half-electron approximation for open shells systems.

Let us now consider the fictitious disproportionation reaction:

$$
\mathrm{M}^{+}+\mathrm{M}^{+} \Rightarrow \mathrm{M}^{2+}+\mathrm{M}^{0}
$$

where both molecules are isolated from each other. In a quantum cell model with a single site orbital (immaterial) per molecule with similar site energies whatever the charge of the molecule, such a process creates a doubly occupied site orbital on $\mathrm{M}^{0}$, and it is clear that the energy change for this reaction only reflects the electrostatic interaction between two electrons on the site orbital of $M^{0}$. We thus write the energy change $\Delta E$ related to this reaction as:

$$
\Delta E=U
$$

where $U$ is the on-site repulsion.

The value obtained for $U$ from the difference between the total energies of the monomer in the distinct charge states are given in the last line of Table I. This calculated $U$ is $4.64 \mathrm{eV}$. On 
the other hand, had $U$ be formally defined as the coulombic repulsion integral for two electrons on the same site orbital $\chi_{p}$ of $\mathrm{TTF}^{0}$, and identifying $\chi_{p}$ to the molecular HOMO:

$$
U=\left\langle\chi_{p} \chi_{p}\left|1 / r_{\mu v}\right| \chi_{p} \chi_{p}\right\rangle
$$

we would get $U=5.32 \mathrm{eV}$. This difference is a clear evidence of the impact of the orbital relaxation: extracting $U$ from (5) allows us to take into account the self-consistent adaptation of the whole electronic cloud to the change in the molecular charge, whereas (6) does not.

3.2. Dimers. - Let us now consider two interacting neighbouring TTFs in a crystal. The two configurations involved in our calculation are related to a doubly charged dimer: $\mathrm{TTF}^{+} \mathrm{TTF}^{+}$ and $\mathrm{TTF}^{2+} \mathrm{TTF}^{0}$. These are involved in a charge transfer (CT) process that strongly modifies the electrostatic potential and should intervene in the high energy CT bands. The disprorportionation reaction (5) may now effectively be seen as a charge transfer process between first neigbhour molecules. In a similar way to that used for the monomers, the energy difference between the two configurations in (5) may be related to changes in the electrostatic energy of the configurations in the now extended Hubbard model. Introducing the repulsion $V$ between two electrons singly occupying neighbouring site orbitals, we get:

$$
\Delta E=U-V .
$$

From the $\Delta E$ calculated from total energy differences involving all valence electrons of the dimer and using the $U$ value of Table I, we then obtain $V=3.05 \mathrm{eV}$. The $V$ value determined without allowing for an in-situ relaxation of the molecular orbitals (i.e. by injecting directly the MO's obtained on the monomers) is $V=2.95 \mathrm{eV}$, which is within $4 \%$ of the previous value.

3.3. TETRAmERs. - - It is of uppermost importance of course to check whether the cluster size has any sizeable effect on these results. We consequently investigated 4 TTF clusters. In this case, it is possible to study the main transfer processes with a total electron population corresponding to the mean band filling, 1 e. $\mathrm{TTF}_{4}^{2+}$. There is an inversion center in the middle of this cluster, so that the relevant symmetry inequivalent optimized configurations are:

$$
\begin{array}{ll}
\mid A>=M^{+} M^{0} M^{0} M^{+} & \mid B>=M^{0} M^{0} M^{+} M^{+} \\
\mid C>=M^{+} M^{0} M^{+} M^{0} & \mid D>=M^{0} M^{+} M^{+} M^{0} \\
\mid E>=M^{0} M^{0} M^{2+} M^{0} & \mid F>=M^{0} M^{0} M^{0} M^{2+}
\end{array}
$$

We give in Table II the corresponding total energies for each configuration. We also give in parentheses the values determined without allowing for an in-situ relaxation of the molecular orbitals. We also give in the same table the corresponding energies expressed in an extended (to all neighbours) Hubbard model (Eq. (4)).

Considering configurations $|E\rangle$ and $|F\rangle$, which have the same Hubbard energy $3 U$, but clearly different VB/HF total energies, evidences the inadequacy of the Hubbard model to relate the configurations energies to a proper combination of $U$ and $V$ terms in a consistent manner. The same conclusion is reached in an attempt to extract the $V_{1}$ term: within the Hubbard Hamitonian, it should be identical by using the energies of the configurations $|E\rangle$ and $|D\rangle$ or the energies of the configurations $|F\rangle$ and $|B\rangle$. The results definitively show an effect of the environment: the configuration energies depend on the local environment because the $\mathrm{VB} / \mathrm{HF}$ energy evaluation procedure takes into account implicitly the charge of the molecules surrounding the dimer under investigation. The $V_{1}$ term extracted from the configurations $\mid D>$ and $\mid E>$ is equal to $3.07 \mathrm{eV}$ : it is thus very close from the dimer values because the $\mathrm{M}^{+} \mathrm{M}^{+}$dimer in $\mid D>$ is symmetrically surrounded by neutral molecules. On the contrary, 
Table II. - Results on the tetramers: $T T F_{4}^{2+}:$ total energies $E$ (in eV) of the configurations $\mid A>$ to $|F\rangle$. E $E_{\mathrm{H}}$ give the corresponding expressions for the extended Hubbard Hamiltonian (see $E q$. (4)). $E_{\Delta}$ give the corresponding expressions for the Hamiltonian defined in equation (8). The intersite terms $V_{2 j}$ and $\Delta_{2}$ of equation (4) and (8) are noted by $V_{k}$ and $\Delta_{k}$ respectively with $k=|i-j|$ denotes the $k^{\text {th }}$ neighbour. The total energies calculated without relaxation of the $M O$ are given in parenthesis.

\begin{tabular}{|l|l|l|l|}
\hline configurations & $E$ & $E_{\mathrm{H}}$ & $E_{\Delta}$ \\
\hline $\mid A>=\mathrm{M}^{+} \mathrm{M}^{0} \mathrm{M}^{0} \mathrm{M}^{+}$ & $\begin{array}{l}-6382.946 \\
(-6382.899)\end{array}$ & $2 U+V_{3}$ & $\begin{array}{l}2 U+V_{3}+20 \Delta_{1} \\
+12 \Delta_{2}+4 \Delta_{3}\end{array}$ \\
\hline $\mid B>=\mathrm{M}^{0} \mathrm{M}^{0} \mathrm{M}^{+} \mathrm{M}^{+}$ & $\begin{array}{l}-6381.537 \\
(-6381.415)\end{array}$ & $2 U+V_{1}$ & $\begin{array}{l}2 U+V_{1}+18 \Delta_{1} \\
+12 \Delta_{2}+6 \Delta_{3}\end{array}$ \\
\hline $\mid C>=\mathrm{M}^{+} \mathrm{M}^{0} \mathrm{M}^{+} \mathrm{M}^{0}$ & -6382.630 & $2 U+V_{2}$ & $\begin{array}{l}2 U+V_{2}+18 \Delta_{1} \\
+12 \Delta_{2}+6 \Delta_{3}\end{array}$ \\
\hline $\mid D>=\mathrm{M}^{0} \mathrm{M}^{+} \mathrm{M}^{+} \mathrm{M}^{0}$ & -6381.763 & $2 U+V_{1}$ & $\begin{array}{l}2 U+V_{1}+16 \Delta_{1} \\
+12 \Delta_{2}+8 \Delta_{3}\end{array}$ \\
\hline $\mid E>=\mathrm{M}^{0} \mathrm{M}^{0} \mathrm{M}^{2+} \mathrm{M}^{0}$ & -6380.197 & $3 U$ & $3 U+16 \Delta_{1}$ \\
& $(-6379.905)$ & & $+12 \Delta_{2}+8 \Delta_{3}$ \\
\hline $\mid F>=\mathrm{M}^{0} \mathrm{M}^{0} \mathrm{M}^{0} \mathrm{M}^{2+}$ & -6379.724 & $3 U$ & $\begin{array}{l}3 U+20 \Delta_{1} \\
+12 \Delta_{2}+4 \Delta_{3}\end{array}$ \\
\hline
\end{tabular}

the dimer $\mathrm{M}^{+} \mathrm{M}^{+}$is located on the cluster edge of $\mid B>$ and the energy is higher than in the preceding case and the corresponding $V_{1}$ is significantly smaller with a value of $2.83 \mathrm{eV}$.

To summarize, an electrostatic model of repulsion between net charges is not sufficient to describe the change in the total energies when a charge (one charge $2+$ on one site or two charges + on neighbouring sites) moves along a neutral chain. This phenomenon is well known in quantum chemistry and is related to what is called "penetration" effect [18].

We then define $\Delta_{23}$ as the penetration integral between molecule $i$ and molecule $j$. This term embodies the difference between the electron-electron repulsion and the electron-core attraction. It is expected to be rather small and to decrease steeply with the intermolecular distance, since for long distance interactions, both electron-electron and core-electron repulsions reduce to point charge interactions. The Hubbard Hamiltonıan modified along these lines then reads:

$$
\begin{aligned}
\hat{H}= & -\sum_{\imath, \jmath, \sigma} t_{\imath \jmath}\left(c_{\imath, \sigma}^{+} c_{\imath+1, \sigma}+\text { h.c. }\right)+\frac{1}{2} \sum_{\imath} U_{\imath} n_{\imath}\left(n_{\imath}-1\right) \\
& +\frac{1}{2} \sum_{\imath \neq \jmath} V_{\imath \jmath}\left(n_{\imath}-Z_{\imath}\right)\left(n_{\jmath}-Z_{\jmath}\right)+\frac{1}{2} \sum_{\imath \neq \jmath} \Delta_{\imath \jmath}\left(n_{\imath} Z_{\imath}+n_{\jmath} Z_{\jmath}\right)
\end{aligned}
$$

We have assumed that the terms $V_{\imath \jmath}$ are identical for electron-electron and core-core interactions, which may not be true but the core-core interaction is a constant in this type of Hamiltonian, merely inducing a global shift of the energy spectrum.

The last column of Table II collects the corresponding formulations $E_{\Delta}$ taking into account the different terms $U_{\imath}, V_{\imath \jmath}$ and $\Delta_{\imath \jmath}$ of equation (8). The extracted $V_{\imath \jmath}$ values are unique whatever the configurations considered. The edge effects are now properly taken into account and have no influence on the values of the parameters. Assuming that the penetration integrals are negligible beyond second neighbours ( $\Delta_{3}=0$ in Eq. (8)), the comparison of AM1 energy 
differences (relative to the lowest total energy $E_{A}$ ) leads to the following values of the parameters (in $\mathrm{eV}$ ):

$$
V_{1}=3.08 ; \quad V_{2}=1.97 ; \quad V_{3}=1.42 ; \quad \Delta_{1}=0.12
$$

while the corresponding values determined without allowing for an in-situ relaxation of the molecular orbitals are:

$$
V_{1}=2.95 ; \quad V_{2}=1.82 ; \quad V_{3}=1.29 ; \quad \Delta_{1}=0.09
$$

The $V_{1}$ values determined in both ways are identical to the dimer values of $3.05 \mathrm{eV}(2.95 \mathrm{eV})$ and this result is a strong evidence of the transferability of the parameters.

3.4. HeXamers. - - The extension of the results to $6 \mathrm{TTF}$ clusters $\mathrm{TTF}_{6}^{3+}$ is straightforward and it is not detailed here. The parameters issued from such a calculation are the following ones:

$$
V_{1}=3.10 ; \quad V_{2}=2.02 ; \quad V_{3}=1.49 ; \quad V_{4}=1.18 ; \quad V_{5}=0.97 ; \quad \Delta_{1}=0.13 ; \quad \Delta_{2}=0.035
$$

while the corresponding values determined without allowing for an in - situ relaxation of the molecular orbitals are:

$$
V_{1}=2.95 ; \quad V_{2}=1.82 ; \quad V_{3}=1.29 ; \quad V_{4}=0.99 ; \quad V_{5}=0.81 ; \quad \Delta_{1}=0.10 ; \quad \Delta_{2}=0.025
$$

These values confirm the conclusions reached in the previous paragraph about the adequacy of the method to extract reliable parameters from chain clusters calculations.

It is noteworthy to remark that these results imply that the polarization of the MOs induces a rather sizeable effect which is almost independent of the type of neighbour and which raise to about $0.2 \mathrm{eV}$.

We present in Figure 1 the variation of $V$ as a function of the distance $R$ between two TTF molecules. The calculation of the $V$ terms was performed following different approaches. Using the above results on $\mathrm{TTF}_{6}^{3+}$, it is possible to extract $V$ up to the $5^{\text {th }}$ neighbour (the limitation to larger distances is directly related to the size of the cluster that can be handled by AMPAC and to our computer facilities). A comparison is made in Figure 1 between the parameters obtained from the non-optimized MOs and from the optimized MOs. It is also possible and fruitful to extract $V$ for all neighbours interactions, from dimer only calculations: the molecules on the dimer are then located at a distance $R$. Finally, for large $R, V$ may be simply calculated as the interaction between point charges. Were the $V$ terms expressing an electrostatic repulsion, they should follow the Coulomb law for large distances. The Coulomb potential, noted $V_{\mathrm{C}}$, is then ( $V_{\mathrm{C}}$ is expressed in $\mathrm{eV}$ and $R$ in $\AA$ ):

$$
V_{\mathrm{C}}=\frac{14.394}{R}
$$

Figure 1 shows that the calculated $V_{k}$ do not all behave at long distance as $V_{\mathrm{C}}$. The agreement stands for the values deduced from dimer calculations and for the values obtained from hexamer results using non-optimized MOs. This result means that the parameter extraction using the Hamiltonian described by equation (8) is fully size consistent when MOs optimized on monomer are used. There thus exists a model Hamiltonian which properly describes the full set of Valence Bond configurations. Therefore, in the Hilbert space spanned by these VB functions, the diagonal elements of the Hamiltonian matrix may be properly calculated within a simple quantum cell model reminiscent of the Pariser-Parr-Pople model, extended to include 


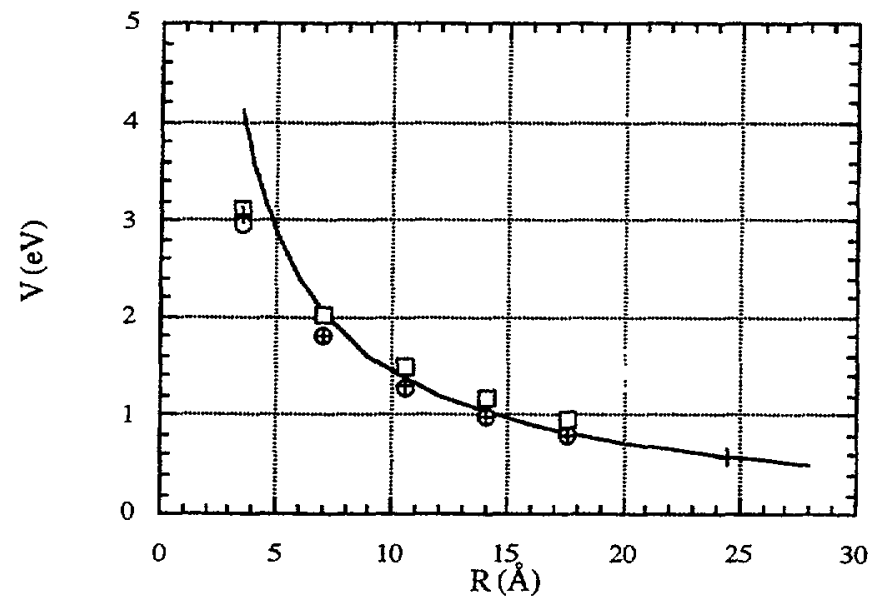

Fig. 1. - Variation of $V(\mathrm{eV})$ as a function of the distance $R(\AA)$ between two TTF molecules. The squares and the circles correspond to the results extracted from calculations on $\mathrm{TTF}_{6}^{3+}$ with and without MO optimization respectively. The crosses correspond to dimer calculation with MO optimization. The solid line represents the Coulomb potential.

penetration interactions. It is also remarkable that coulombic repulsion terms may be evaluated from dimer calculations.

On the contrary, the $V$ values deduced from hexamer results using optimized MOs show a small departure from the Coulomb law at long distance (the difference is about $0.15 \mathrm{eV}$ ). The effective Hamiltonian is thus not suited for a proper extraction of electrostatic interactions in this case. The full optimization of the MOs in each configuration yields an extra energetic term which depends on the nature of the configuration itself. It results in a decrease of the total cluster energy which is maximum for the configurations $|E\rangle$ and $\mid F>$ of Table II, i.e. when there are doubly charged molecules in the cluster: the relaxation of the molecular orbitals is more effective in the more ionic configuration.

3.5. Parameters of ORganic Salts. - In order to discuss more precisely the absolute values of the electrostatic parameters, these were calculated for different molecules all derived from the tetrathiafulvalene molecule (TTF): tetramethyl TTF (TMTTF), Bis Ethylene-dithia TTF (BEDTTTF) and Dimethyl Ethylene-dithia TTF (DIMET). The atomic coordinates are taken from crystallographic structures: $\mathrm{TMTTF}_{2} \mathrm{PF}_{6}$ measured at $4 \mathrm{~K}$ and 1 atm [19], $\beta-\mathrm{BEDTTTF}_{2} \mathrm{I}_{3}$ at $4.5 \mathrm{~K}$ under a pressure of $P=1.5 \mathrm{kbar}[20], \mathrm{DIMET}_{2} \mathrm{SbF}_{6}$ at $300 \mathrm{~K}$ and $1 \mathrm{~atm}$ [21]. These salts are representative of quasi-one and quas1-two dimensional structures for $\mathrm{TMTTF}_{2} \mathrm{PF}_{6}$ and $\beta-\mathrm{BEDTTF}_{2} \mathrm{I}_{3}$ respectively, while the $\mathrm{DIMET}_{2} \mathrm{SBF}_{6}$ salt evidences a quasi-1D structure but with a large dimerization of the intrastack transfer integrals as discussed previously [12].

3.6. Monomers. - Table III shows the total energies obtained for the neutral $\mathrm{M}^{0}$, monocationic $\mathrm{M}^{+}$and dicationic $\mathrm{M}^{2+}$ species which are used to calculate $U$. The total energies were calculated by means of the AM1 semi-empirical approximation. The corresponding $U$ values (see Eq. (5)) are given in the penultimate line of Table III. They range around $4.1 \mathrm{eV}$ and expectedly decrease with the size of the molecule. They are twice as large as previous results, obtained using the half-electron CNDO calculation [12]. 
Table III. - Same entries as Table I for molecule M.U (ab inztio) were determined from total energy differences calculated by means of the GAUSSIAN 92 package [Gaussian 92, Revision D.2, Frisch M.J., Trucks G.W., Head-Gordon M., Gill P. M.W., Wong M.W., Foresman J.B., Johnson B.G., Schlegel H.B., Robb M.A., Replogle E. S., Gomperts R., Andres J.L., Raghavachari K., Binkley J.S., Gonzalez C., Martin R.L., Fox D.J., Defrees D.J., Baker J., Stewart J.J.P., and Pople J.A., Gaussian, Inc., Pittsburgh PA, 1992].

\begin{tabular}{|l|c|c|c|}
\hline $\mathrm{M}$ & TMTTF & BEDTTTF & DIMET \\
\hline$E\left(\mathrm{M}^{0}\right)$ & -2222.951 & -2944.761 & -2583.398 \\
\hline$E\left(\mathrm{M}^{+}\right)$ & -2216.216 & -2937.951 & -2576.576 \\
\hline$E\left(\mathrm{M}^{2+}\right)$ & -2205.071 & -2927.244 & -2565.635 \\
\hline$I_{1 \mathrm{t}}\left(\mathrm{M}^{0}\right)$ & 6.73 & 6.81 & 6.82 \\
\hline$I_{1 \mathrm{~K}}\left(\mathrm{M}^{0}\right)$ & 7.12 & 7.27 & 7.23 \\
\hline$I_{1 \mathrm{t}}\left(\mathrm{M}^{+}\right)$ & 11.15 & 10.71 & 10.94 \\
\hline$I_{1 \mathrm{~K}}\left(\mathrm{M}^{+}\right)$ & 11.57 & 11.23 & 11.41 \\
\hline$U$ & 4.41 & 3.90 & 4.12 \\
\hline$U(a b$ initio $)$ & 4.99 & 4.61 & 4.78 \\
\hline
\end{tabular}

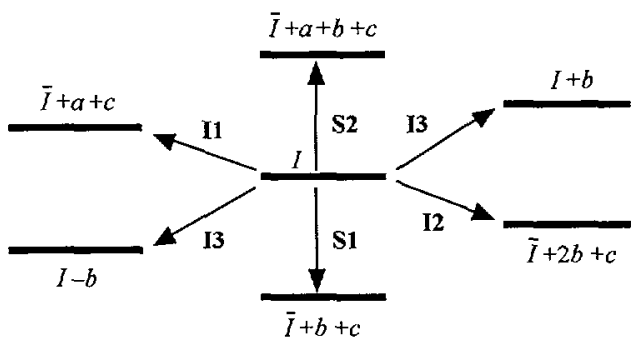

Fig. 2. - Schematic representation of the TMTTF packing in the ( $a . b)$ plane. Symmetry operations from the central TMTTF (labelled $I$ ) are indicated. The zig-zag structure of the chain imposes that both $I$ and $\bar{I}+b+c$ molecules belong to the unt cell so that the intrachain interactions, noted $S 1$ and $S 2$, are inequivalent. The interchain interactions are noted $I 1, I 2$ and $I 3$. Equivalent pictures also apply to the definition of the interactions in $\beta-\mathrm{BEDTTF}_{2} \mathrm{I}_{3}$ and $\mathrm{DIMET}_{2} \mathrm{SbF}_{6}$.

The last line of the table collects the values of $U$ obtained from $a b$ initio Hartree-Fock calculations using a double-zeta $4-31 \mathrm{G}$ basis set. The $a b$ initio $U$ range around $4.8 \mathrm{eV}$, which is somehow larger than the AM1 results but not qualitatively different. The variation of $U$ with the size of the molecule shows the same trend. Moreover, the ratio $U$ (AM1) / $U$ ( $a b$ initro) is the same for each molecule. These $a b$ initio results sustain the use of a semi-empirical approach for determining the correlation parameters.

The AM1 or ab initio purely molecular $U$ terms may appear far too large compared to estimations from experımental data [22-24]. This point will be clarified in following sections.

3.6.1. Dimers. - Dimer calculations were performed for the three structures: $\mathrm{TMTTF}_{2} \mathrm{PF}_{6}$, $\beta-\mathrm{BEDTTTF}_{2} \mathrm{I}_{3}$ and $\mathrm{DIMET}_{2} \mathrm{SbF}_{6}$, and all types of first neighbour interaction. In Figure 2, we figure out the distinct labels associated with the first neighbour interactions. $S_{\imath}$ or $I_{2}$ stand for the intrastack and interstack interactions respectively. The $V$ values which are collected in Table IV are obtained from equation (4) using the $U$ values of Table III. 
Table IV. - Results on the dimers $M_{2}^{+}: \Delta E$ are the differences between the total energies of the $\left[M^{+} M^{+}\right]$configurations and the $\left[M^{2+} M^{0}\right]$ configurations, and $V$ is the interaction between first neighbours (all entries in eV).

\begin{tabular}{|lr|l|l|l|l|l|}
\hline Interaction & & $S_{1}$ & $S_{2}$ & $I_{1}$ & $I_{2}$ & $I_{3}$ \\
\hline TMTTF & $\Delta E$ & 1.50 & 1.53 & 2.49 & 2.20 & 2.46 \\
& $V$ & 2.91 & 2.88 & 1.92 & 2.21 & 1.95 \\
\hline \multirow{2}{*}{ BEDTTTF } & $\Delta E$ & 1.48 & 1.78 & 1.78 & 1.81 & 1.73 \\
& $V$ & 2.41 & 2.11 & 2.11 & 2.08 & 2.16 \\
\hline DIMET & $\Delta E$ & 1.31 & 1.87 & 2.05 & 2.15 & 1.95 \\
& $V$ & 2.80 & 2.24 & 2.06 & 1.96 & 2.16 \\
\hline
\end{tabular}
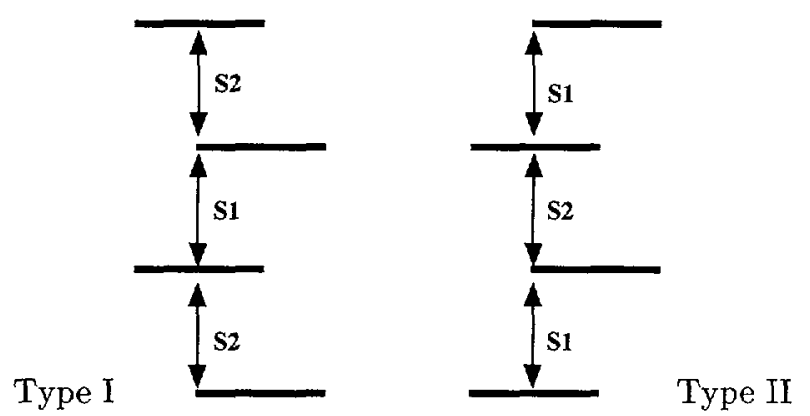

Fig. 3. -- Schematic representation of the TMTTF chain showing the two possible geometries for a tetramer.

The calculated $V$ values range roughly between 2 and $3 \mathrm{eV}$, i.e. of the order of $U / 2$. They are closely related to the crystal structure, but in a different way than the transfer integrals do. The $V$ terms do reproduce the dimensionality of the crystal: for the quasi-1D $\mathrm{TMTTF}_{2} \mathrm{PF}_{6}$ salt, the intradimer $V_{S 1}$ and interdimer $V_{S 2}$ terms are $1 \mathrm{eV}$ larger than any other interstack $V_{I}$ term. The 2D BEDTTTF results show that the intradimer term $V_{S 1}$ is the largest one, but it is only $0.3 \mathrm{eV}$ higher than the other terms. The case of DIMET is intermediate: $V_{S 1}$ is the largest one, the $V_{I 2}$ are equivalent and similar to the TMTTF case, while the interdimer interaction, $V_{S 2}$, is intermediate between the two sets.

The average value $\Delta E$ is now around $1.8 \mathrm{eV}$ which is of the same order of magnitude as the visible CT bands observed experimentally [22-24]. Obviously, the calculated values do not correspond exactly to the energy differences between stationnary states that are in fact VB mixtures of the different configurations. The interaction energy between the two configurations - which we could call the transfer integral - is therefore also involved in the CT band. Nevertheless it is admitted that such transfer corrections are rather weak in molecular crystals, due to the weak overlap between the MO's on neighbourng molecules [13], and our calculated transition energies thus should not be very different from the true theoretical absorption band.

3.6.2. Tetramers. - The tetramers calculations were performed only in the case of the intrachain interaction corresponding to the crystal geometry of $\mathrm{TMTTF}_{2} \mathrm{PF}_{6}$. Taking into account the real crystal structure leads to two possible choices for the tetramer $\mathrm{TMTTF}_{4}^{2+}$, illustrated by the two different interaction sequences shown in Figure 3.

We give in Table $\mathrm{V}$, the corresponding total energies for each configuration. Again, the total energies using the molecular orbitals optimized on the isolated monomers are given in 
Table V. - Results on the tetramers $\operatorname{TMTTF}_{4}^{2+}$ : total energies $\mathrm{E}$ of the configurations $\mid A>$ to $|F\rangle$ (the values corresponding to non-optimized MOs are given in parenthesis). $E_{\Delta}$ give the expressions for the model Hamiltonian (see Eq. (8); $\Delta_{3}$ terms were omitted). For each configuration, the first line corresponds to the type I cluster, and the second line to the type $I I$ cluster of Figure 3.

\begin{tabular}{|l|l|l|}
\hline & $E$ & $E_{\Delta}$ \\
\hline $\mid A>$ & $-8875.835(-8875.768)$ & $2 U+V_{3}(\mathrm{I})+20 \Delta_{1}+12 \Delta_{2}$ \\
$\mathrm{M}^{+} \mathrm{M}^{0} \mathrm{M}^{0} \mathrm{M}^{+}$ & $-8875.785(-8875.713)$ & $2 U+V_{3}(\mathrm{II})+20 \Delta_{1}+12 \Delta_{2}$ \\
\hline $\mid B>$ & $-8874.648(-8874.477)$ & $2 U+V_{S 2}+18 \Delta_{1}+12 \Delta_{2}$ \\
$\mathrm{M}^{0} \mathrm{M}^{0} \mathrm{M}^{+} \mathrm{M}^{+}$ & $-8874.579(-8874.407)$ & $2 U+V_{S 1}+18 \Delta_{1}+12 \Delta_{2}$ \\
\hline $\mid C>$ & $-8875.567(-8875.430)$ & $2 U+V_{2}+18 \Delta_{1}+12 \Delta_{2}$ \\
$\mathrm{M}^{+} \mathrm{M}^{0} \mathrm{M}^{+} \mathrm{M}^{0}$ & $-8875.526(-8875.384)$ & $2 U+V_{2}+18 \Delta_{1}+12 \Delta_{2}$ \\
\hline $\mid D>$ & $-8874.855(-8874.629)$ & $2 U+V_{S 1}+16 \Delta_{1}+12 \Delta_{2}$ \\
$\mathrm{M}^{0} \mathrm{M}^{+} \mathrm{M}^{+} \mathrm{M}^{0}$ & $-8874.843(-8874.618)$ & $2 U+V_{S 2}+16 \Delta_{1}+12 \Delta_{2}$ \\
\hline $\mid E>$ & $-8873.400(-8872.984)$ & $3 U+16 \Delta_{1}+12 \Delta_{2}$ \\
$\mathrm{M}^{0} \mathrm{M}^{0} \mathrm{M}^{2+} \mathrm{M}^{0}$ & $-8873.357(-8872.947)$ & $3 U+16 \Delta_{1}+12 \Delta_{2}$ \\
\hline $\mid F>$ & $-8872.880(-8872.619)$ & $3 U+20 \Delta_{1}+12 \Delta_{2}$ \\
$M^{0} M^{0} M^{0} M^{2+}$ & $-8872.845(-8872.579)$ & $3 U+20 \Delta_{1}+12 \Delta_{2}$ \\
\hline
\end{tabular}

parentheses; in this way we can estimate the influence of the environment on the energy and orbital relaxation. We also give in the same table the corresponding energies expressed in an extended (to all neighbours) Hubbard model. The calculations were performed for the two clusters I and II defined in Figure 3. With $U=4.41 \mathrm{eV}$, the $V$ parameters take the following values (in $\mathrm{eV}$ ):

$$
V_{S 1}=2.96 ; \quad V_{S 2}=2.92 ; \quad V_{2}=1.98 ; \quad V_{3}(I)=1.46 ; \quad V_{3}(I I)=1.47 ; \quad \Delta_{1}=0.13
$$

while the corresponding values determined without allowing for an in-situ relaxation of the molecular orbitals are:

$$
V_{S 1}=2.77 ; \quad V_{S 2}=2.74 ; \quad V_{2}=1.79 ; \quad V_{3}(I)=1.26 ; \quad V_{3}(I I)=1.28 ; \quad \Delta_{1}=0.09
$$

As expected from the above discussion on TTF clusters, the $V_{S 1}$ values are close to the ones extracted from dimer calculations. Their decrease relative to the TTF values reflects the difference in the corresponding $U$ values. The variation of $V$ with the intermolecular distance follows the Coulomb potential law as already discussed. As an illustration, the only slight dimerization of the stack in $\mathrm{TMTTF}_{2} \mathrm{PF}_{6}$ does not induce a significative difference between $V_{3}(I)$ and $V_{3}(I I)$.

From the energies of the tetramer configurations, one may get some insight into the charge transfer (CT) spectrum by looking at the possible CT excitations involving neighbouring molecules. The corresponding excitations energies may be also expressed as a function of the electrostatic terms entering the definition of equation (8). Figure 4 collects the results obtained along these lines. We do not have access yet to the true theoretical CT bands. since we are only considering in this work VB structures instead of exact stationary states. The intensities of the band are also beyond the scope of this paper.

Two main groups of spectral lines are found: the first one involves CT energies around $4000 \mathrm{~cm}^{-1}$ and corresponds roughly to the $V$ terms; the second group involves both $U$ and $V$ and is around $13000 \mathrm{~cm}^{-1}$ These results qualitatively agree with the experimental spectra of 

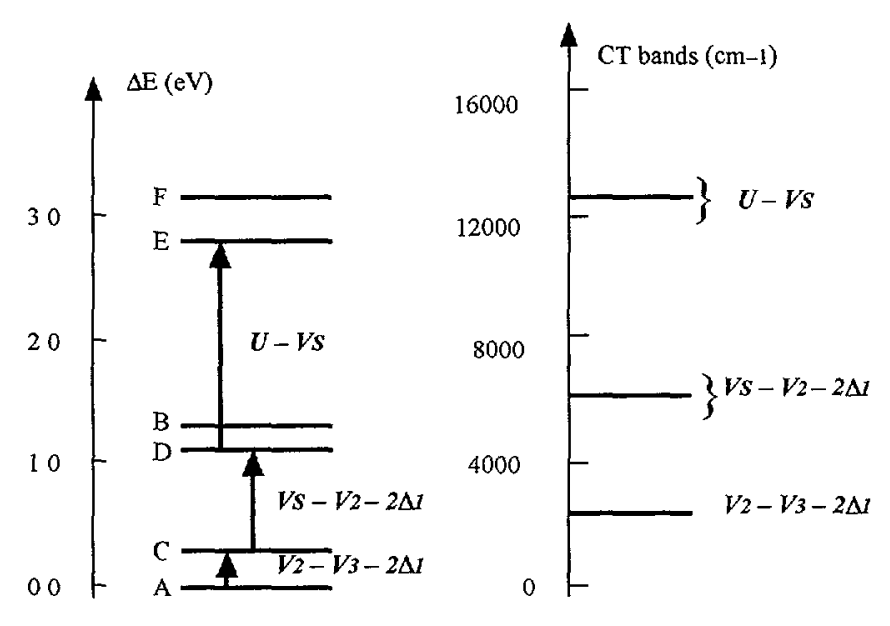

Fig. 4. - Schematic representation of the CT bands in TMTTF chains. The left part of the figure shows the energies of the Valence Bond configurations $\mid A>$ to $\mid F>$ relative to the energy of $|A\rangle$ (see Tab. $V$ for definition). The possible charge transfers between these states are also indicated in the framework of the Hamltonian described in equation (8). The right part of the figure is a crude representation of the theoretical spectrum. The effect of stack dimerization on $V_{S}$, which leads to a doubling of the CT bands at $U-V_{S 1}\left(V_{S 2}\right)$ and $V_{S 1}\left(V_{S 2}\right)-V_{2}-2 \Delta_{1}$ is not represented for clarity.

organic salts which evidence a band called " $\mathrm{B}$ " whose energy is larger than $1 \mathrm{eV}$ and a "A" band in the domain 0.25 to $0.40 \mathrm{eV}$ [23].

\section{Conclusion}

In order to size up the relative magnitude of the coulombic interactions between conduction electrons in organic conductors, we set up a mixed Valence-Bond/Hartree-Fock method applied to all valence electrons calculations on finite size clusters. The hierarchy of energy levels associated to the relevant set of electronic configurations of the clusters was analysed with an effective Hamiltonian built upon a single site orbital per molecule and thus comparable to model Hamiltonıans such as the (extended) Hubbard or Pariser-Parr-Pople Hamiltonıans. The extraction of this effective Hamiltonıan yields a Pariser-Parr-Pople like Hamiltoman, extended to include penetration interactions. Moreover, it appears that it is most consistent when MOs optimized on monomer are used; the effective Hamiltonian reproducing the whole set of VB energy levels. Therefore, in the Hilbert space spanned by these VB functions, the diagonal elements of the Hamiltonian matrix may be properly calculated within a simple quantum cell model.

The effective on-site repulsion $U$ extracted in this way are about $4 \mathrm{eV}$ while the intersite $V$ repulsion terms range between 2 and $3 \mathrm{eV}$ for first-neighbour interactions and they follow a Coulomb law for large intermolecular distances. Although these terms may each appear too large compared to previous estimations [22-24], the roughly calculated CT excitations are still in good agreement with the experimental results which only rely on energy differences. Recent proposals for a determination of these parameters on the basis of reflectivity measurements $[10 \mathrm{~b}]$ would essentially lead to the same range of parameters as in [22-24], because the analysis of the experimental results depends on the type of the model one uses, i.e. the extended Hubbard model restricted to first-neighbour interactions. We must state here that there is no inconsistency between our results and the values commonly quoted in the literature. 
As mentioned above, this work concentrated on the extraction of the diagonal part of the effective Hamiltonian. It remains to set up a similar analysis for deriving the off-diagonal part of the effective Hamiltonian which essentially relies on the charge transfer processes between neighbouring molecules. Matrix elements between the various VB structures now involve non orthogonal molecular orbitals: the MOs are orthogonal in each VB structure, but the MOs of a given molecule in a given charge state are not orthogonal anymore to those of the same molecule in another charge state. The off diagonal part of the Hamiltonian matrix will therefore require much more efforts than the tight-binding techniques for calculating transfer integrals. It must also be emphasized that one should take great care of the counter-anions potential, which for symmetry reasons might strongly contribute to the transfer terms. We assumed in this work that the anions could be discarded for comparing the various charge distributions in the organıc stacks. Indeed, if the electronic clouds of the anions and organic molecules were optimized on monomers, the anın-cation interactions, for symmetry reasons, would be simply additive and give essentially the same contribution for each VB configuration. The difference in the cation MOs would merely induce a slight correction in the coulombic terms, similar to the $\mathrm{D}$ correction. The anions should nevertheless be taken into account if one has to deal with possible effective interaction mechanisms involving the differential polarization of the molecules and anions in distinct electronic configurations.

Such considerations raise many methodological questions about the most appropriate way of setting up calculation schemes that would give the clearest interpretation of the relevant physical phenomena in these materials. VB inspired or not, variational or perturbational, such schemes always rely on some zeroth order stage. Any theoretical analysis is therefore strongly dependent on the choice of this zeroth order reference. For example, had we imposed a unique set of MOs for all VB configurations, we would have obtained a different effective Hamiltonian with larger interaction parameters; an extensive Configuration Interaction (CI) or perturbation scheme being necessary to reach the same level of accuracy as the present VB calculation. In the same way, one should ask whether the MOs should be optimized in-situ or not, the second alternative being obviously less accurate but much more simply analysable. In the prospect of dealing with sizeable two dimensional clusters, it seems more reasonable to employ monomer optimized MOs; the results of this work showing no great differences in terms of the order of magnitude of the coulombic parameters. The understanding of the effect of the full optimization of the MOs (projected in our zeroth order space) would allow for the inclusion of new polarization terms in the effective Hamiltonian.

The magnitude of the coulombic terms is found to differ qualitatively from previous estimations and evidences the long range part of the potential, which has sometimes been advocated in the literature [25]. It is moreover of first importance to analyze the solutions of extended Parser-Parr-Pople Hamiltoman, within the determined parameter range, and to check whether or not these solutions may alternatively be attained by means of a more usual (extended) Hubbard Hamiltonian.

\section{References}

[1] Jérome D., Mazaud A., Ribault M. and Bechgaard K., J. Phys. Lett. France 41 (1980) L95-98.

[2] Wang H.H., Carlson K.D., Geiser U., Kini, A.M., Schultz A.J., William J.M., Montgomery L.K., Kwok W.K., Welp U., Vandervoort K.G., Schriber J.E., Overmyer D.L., Jung D., Novoa J.J. and Whangbo M.-H., Synth. Metals 42 (1991) 1983-1990. 
[3] Proceedings of the International Conference on Science and Technology of Synthetic Metals, Seoul (Korea) Synth. Metals 70 (1995).

[4] Jérome D. and Schulz H.J., Adv. in Physics 31 (1982) 299-490.

[5] Coulon C., in "Organic and Inorganic Low-Dimensıonal Crystallıne Materials", Vol. 168, P. Delhaes and M. Drillon Eds (Plenum Press, NATO ASI Series, 1987) 201-218.

[6] Welp U., Flesher S., Kwok W.K., Crabtree G.W., Carlson K.D., Wang H.W., Geiser U., Williams J.M. and Hitsman V.M., Phys. Rev. Lett. 69 (1992) 840-843.

[7] Delhaes P. and Ducasse L., Localized and Itinerant Molecular Magnetism, NATO ASI Series (Tenerife, Spain, 1995) to appear.

[8] Caron L.G. and Bourbonnais C., Physica B 143 (1986) 453-455.

[9] Emery V.J., Bruinsma R. and Barısic S., Phys. Rev. Lett. 48 (1982) 1039-1043.

[10] a) Mila F. and Zotos X., Europhys. Lett. 24 (1993) 133-138; b) Mila F., Phys. Rev. B 52 (1995) 4788-4793.

[11] Scalapino D.J., Physica C 185-189 (1991) 104-109.

[12] Fritsch A. and Ducasse L., J. Phys. I France 1 (1991) 855-880.

[13] Ducasse L., Abderrabba M., Hoarau J., Pesquer M., Gallois B. and Gaultier J., J. Phys. C 19 (1986) 3805-3820.

[14] Barriquand F. and Sawatzky G.A., Phys. Rev. B 50 (1994) 16649-16667.

[15] AMPAC 5.0, (C) 1994, Semichem, 7128 Summit, Shawnee, KS 66216 (USA).

[16] Demiralp E. and Goddard W.A.III, J. Phys. Chem. 98 (1994) 9781-9785.

[17] Mc Weeny R., Methods of molecular quantum mechanics (Academic Press, 1992).

[18] Pople J. A. and Beveridge D. L., Approximate Molecular Theory (Mc Graw Hill, New York, 1970).

[19] Granier T., Gallois B., Ducasse L., Fritsch A. and Filhol A.. Synth. Metals 24 (1988) 343-356.

[20] Schultz A. J., Wang H.H., William J.M. and Filhol A., J. Am. Chem. Soc. 108 (1986) 7853-786.

[21] Gallo1s B., Gaultier J., Bechtel F., Chasseau D., Hauw C. and Ducasse L., Synth. Metals 19 (1987) 419-424.

[22] For a review see: Jacobsen C.S., J. Phys. C 19 (1986) 5643-5654.

[23] Delhaès P. and Garrigou-Lagrange C., Phase Transitions 13 (1988) 27-33.

[24] Mazumdar S. and Soos Z.G., Phys. Rev. B 33 (1981) 2810-2823.

[25] Emery V.J. and Kivelson S.A., Physica C 209 (1993) 597-621. 